\title{
Field Evaluation of a Mobile Location-Based Notification System for Police Officers
}

\author{
Jan Willem Streefkerk \\ TNO Human Factors \\ Kampweg 5, 3769 DE Soesterberg \\ the Netherlands \\ +31 (0)346356343 \\ j.w.streefkerk@tno.nl
}

\author{
Myra P. van Esch-Bussemakers \\ TNO Human Factors \\ Kampweg 5, 3769 DE Soesterberg \\ the Netherlands \\ +31 (0)346 356457 \\ myra.vanesch@tno.nl
}

\author{
Mark A. Neerincx \\ Man Machine Interaction group \\ Delft Technical University \\ Delft, the Netherlands \\ +31 (0)346 356298 \\ mark.neerincx@tno.nl
}

\begin{abstract}
To increase police officer awareness of incident locations, the Dutch police developed and implemented a location-based notification system (LBNS). This mobile service notifies police officers proactively to warrants, agreements and police focal points in their current vicinity. To assess the accuracy, efficiency, effectiveness and user experience of this service, a longitudinal field evaluation was conducted with thirty police officers over four months. The results show that using the LBNS, police officers were better informed of relevant information in their environment and this led to positive operational results. Users considered the interface clear and easy to use. However, users indicated that the system presented too many or non-relevant notifications and that the system is overly complex. Recommendations for further development of the LBNS are to mitigate unwanted interruption by intelligent filtering of notifications and integration of system components.
\end{abstract}

\section{Categories and Subject Descriptors}

H.5.2 [Information Systems]: User Interfaces - Graphical user interfaces, Evaluation / methodology.

\section{General Terms}

Measurement, Performance, Design, Reliability, Human Factors.

\section{Keywords}

Mobile Computing, Notification Systems, Situation Awareness, Field Testing, Police, Longitudinal Evaluation.

\section{INTRODUCTION}

For mobile police officers, increasingly more information becomes available on surveillance. They have to be aware of locations of incidents and colleagues, crime hotspots and police focal points. Most of this information is only necessary and relevant at specific locations. For example, police officers only have to be aware that a particular location is a criminal hotspot so they can act on this information when they are in the vicinity. In

Permission to make digital or hard copies of all or part of this work for personal or classroom use is granted without fee provided that copies are not made or distributed for profit or commercial advantage and that copies bear this notice and the full citation on the first page. To copy otherwise, or republish, to post on servers or to redistribute to lists, requires prior specific permission and/or a fee. MobileHCI 2008, September 2-5, 2008, Amsterdam, the Netherlands. Copyright (C) 2008 ACM 978-1-59593-952-4/08/09...\$5.00. addition, this information is dynamic in nature, changing over time. New incidents may come up during the day, or colleagues may finish their shift and are no longer available to provide assistance. This entails a need for information support for police officers on the street. Current developments in geographical information systems (GIS) and mobile internet enable locationbased information presentation on mobile devices (e.g. PDA's). Such location-based notification systems (LBNS) present relevant information proactively (i.e. via notifications) based on the current geographical location of the user [7].

The Dutch police designed and implemented a LBNS to support three police procedures that require awareness of incident locations. The system notifies police officers proactively to location-specific police focal points and warrants when they are in the vicinity of such a location. The system was implemented as a geographical map application on a PDA using GPS location tracking. The application employs auditory signals and pop-up screens to notify police officers and facilitates access to operational information on the handheld device. This is expected to help police officers in three ways. First, they are able to handle incidents faster and more effectively. In addition, because they have information available within the use context, officers have to rely less on communication with the emergency room. Finally, their awareness of incident locations will increase when they are notified to relevant information on location. These three effects are expected to have a positive influence on operational results, such as a higher chance of apprehending criminals, or reducing nuisance. However, the effects of this innovative system on police work efficiency and effectiveness and user experience must be carefully evaluated. Potential downsides of a LBNS are distraction or interruption by non-relevant notifications. For example, when a police officer is en-route to a high-priority incident, a notification about a criminal that needs to be apprehended might be distracting. Thus, in evaluating this system, the trade-off between receiving valued information while minimizing non-relevant interruptions needs to be addressed.

This paper reports on a longitudinal field evaluation of the implemented LBNS for police officers. Twenty-six police officers used this system in their daily work for a period of four months. Using information from interviews, observation, questionnaires and log-file analysis, the following research questions were addressed. Does this LBNS support police officers' task efficiency and effectiveness? Does it have a positive influence on the user experience? And finally, how can potential negative effects of LBNS be mitigated? In the following sections, first the system and the police procedures it was designed for are 
described. Then, the method and results of the evaluation are presented. Finally, the implications for further design of this LBNS are specified.

\section{RELATED WORK}

Location based notification systems form a special class of context-aware systems, i.e. by providing a notification based on the users location. Research on context-aware systems has been carried out for quite some time, in a diversity of domains such as firefighting [5], military [8] and first-response [12] but also tourism [2]. Most of these systems were experimental prototypes to support user navigation, notification or background information regarding landmarks (e.g. providing historical information about a church in a city). For example, SeeVT is a location-based notification system that provided background information via notifications on a mobile device for visitors to the Virginia Tech university campus [11].

Location-based systems in critical domains, such as the Land Warrior System for the military, are designed to support soldiers in navigation and situation awareness [8]. This particular system provides information about enemy presence, terrain and landmarks using GPS coordinates. However, it provides as yet no proactive notifications, but instead relies on user "information pull" (e.g. by looking at the map at the appropriate moment).

\subsection{Mobile Police Support Systems}

Another critical domain is the police work environment. Studies here include task and work analyses, sociological studies and field work (e.g. [4]). Several HCI related studies on designing computer support for police officers can be found, however these do not address location-based notification specifically. A requirements analysis of personal mobile computers for police officers indicated useful directions for supporting officers patrolling on foot [1]. For example, mobile computers enable them to record crime information including photographic or video information, and displaying incidents on digital maps. However, the authors conclude that the cognitive cost of using mobile computers while performing other activities should be assessed and mitigated. In a recent related effort, mobile navigation support for police officers on foot was designed [13]. Design elements included indicating hotspots, incidents, surveillance routes on a map and providing notifications. To lower cognitive costs, map manipulations such as zooming and panning were automated based on the priority of the navigation task. Although no field evaluation was performed, it was concluded that automating map manipulations could enhance efficiency of police work.

Notifying police officers to relevant information is also an important aspect in police team awareness systems. These systems facilitate communication and information sharing between officers. An example is the "we-centric" service to support spontaneous communication between police officers [14]. This service enabled police officers to see which of their colleagues was available to provide assistance in handling an incident. In addition, the service indicated which colleague was relevant with respect to the incident (e.g. a colleague who had visited that incident location before was considered highly relevant). Notifications were used to prompt colleagues to contact each other, specifying why that colleague was relevant. A field evaluation of an experimental prototype was planned, and the expectations were that the system would encourage users to share valuable implicit knowledge. Another team awareness system was inspired by an ethnographic police field-study in Sweden. The author proposed the design of an in-car awareness system to support communication between dispatchers and patrolling officers [9]. The display indicated the status of all patrols and their distance to the incident location, based on GPS coordinates. This was expected to support more efficient task allocation and awareness of other patrols but no system evaluation was performed with end-users.

Concluding, research efforts focused on experimental mobile devices to enhance situation awareness, improve task performance and communication between police officers. Up to now, no location-based notification system for police officers has been implemented. Furthermore, only a limited number of prototypes of these systems have been evaluated in the field with end-users. To our knowledge, the current implemented LBNS represents a unique system in the police domain.

\subsection{Field Evaluation of Mobile Systems}

In a field evaluation of mobile systems, the goal is to assess the impact of the system on (teams of) users, work processes and the organization. By evaluating mobile systems within the actual use context, influences of this rich and dynamic context on system use can be measured. This knowledge is subsequently used to adapt the appropriateness of the designs. Usually, a combination of methods and measures is used, such as work process efficiency and effectiveness and user experience measures.

A literature overview showed that studies in the lab are more prevalent for evaluation of these mobile systems [6]. The authors concluded that for usability evaluation, the added value of field research is very little. In contrast, we have recently suggested that in professional domains, field evaluation should be an integral part of designing appropriate systems, because it targets different research questions [10]. Still, care must be taken to select the appropriate methods and measures and tune these to the application domain. In this study, "efficiency" and "effectiveness" are established based on quality criteria specific of police work processes. Furthermore, incorporating end-users leads to valid results and appropriate designs. However, the evaluation is dependent on the availability and participation of these end-users. Their availabilty may be diminished due to busy schedules or scarce resources. In addition, care must be taken not to let the evaluation influence ongoing work too much. For example, using questionnaires or rating scales on the mobile device itself may prevent recall errors later on, but this also distracts users from their tasks [3]. In actual police settings, such distraction incurs risks to the security of officers.

Concluding, there is a lack of empirical field studies of mobile systems with actual end users. The current research aims to show how field evaluation can improve the appropriateness of these innovative mobile systems in critical domains.

\section{LBNS FOR POLICE OFFICERS}

The Dutch police was interested in the potential benefits from location based notification, such as improved officer awareness of their environment and increased chances of apprehending criminals. Their vision was a mobile system that proactively 
notifies police officers on surveillance when they are in the vicinity of a location that requires their attention. This system should support awareness of three types of location-specific information, particularly open warrants (such as fines), agreements on location (such as environmental regulations) and police focal points (such as criminal hotspots).

- Open warrants. These warrants are issued when perpetrators have to pay a sentenced fine or spend time in jail. Police officers need to be aware of these open warrants, so they can visit the address when they are in the vicinity. This increases the chance of apprehending the perpetrator.

- Agreements on location. These are locations where regulations are in effect that specify what is and is not permitted at that location. Examples are regulations about noise pollution or bar opening times. Police officers only need to be aware of these agreements when there is an incident reported at the location. Thus, a notification system does not have to notify them to agreements, unless an incident is reported.

- Police focal points. These focal points are locations with increased criminal activity. For example, when police officers are aware of burglary hotspots, they can be extra vigilant when they are in the vicinity. At the time of the evaluation, this functionality was not yet implemented in the LBNS, so no notifications to police focal points were given.

Note that these three types of locations are not concerned with time-critical incidents, although officers might need to be aware of these locations when handling incidents (such as agreements). In the current situation, police officers have to approach the emergency room via radio transceiver with requests about a specific location. They will ask whether agreements, open warrants or police focal points are in effect at a particular location. Alternatively, they can approach colleagues informally with questions via mobile phone. The final way to get information while on the street is the use of notebooks ("notitieboekjes"). During the morning briefing, police officers write down incidents, addresses, names and other operational information and check their notes when necessary during the day.

Furthermore, police officers work in shifts, making the briefing the main point of information sharing between most (but not all) active officers. Different types of shift can be distinguished, such as emergency response or a "free" shift. During emergency response, officers have less opportunity to respond to non-time critical incidents. Warrants, agreements and police focal points are generally of a lower priority than emergency response incidents. Similarly, officers with specific roles (e.g. a district officer or a youth officer) often have to be more in-depth aware of incident addresses in a specific region.

Current practice shows that officer awareness of these locations is dependent on their own proactivity (e.g. checking their notes at the right time and place). The problem police officers run into is that they are not aware of relevant locations at the right time or place. Furthermore, they are reliant on information from other sources (emergency room, colleagues). Using a proactive, mobile location-based notification system, officer awareness of open warrants and police focal points is expected to increase. Police officers are expected to be more self-reliant and will have more information available in the use context, which will aid their
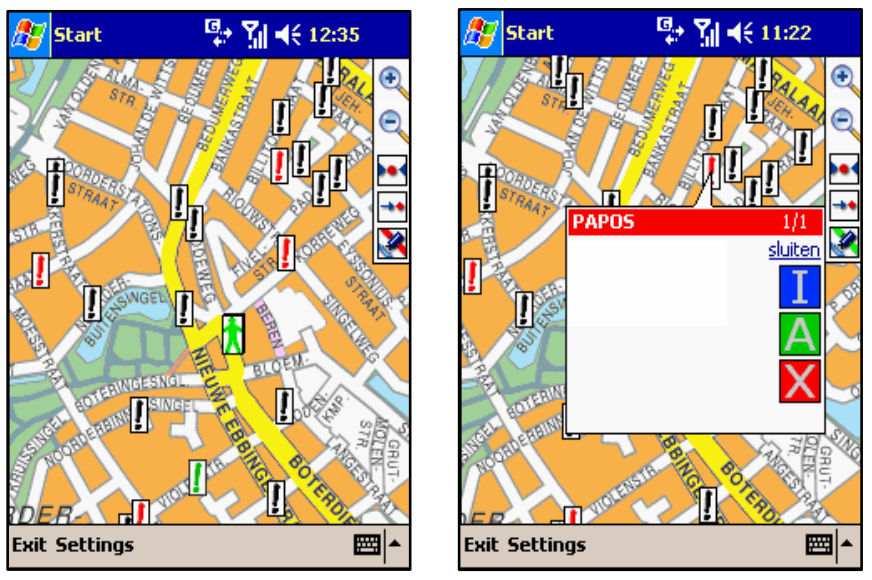

Figure 1. Screenshots from the LBNS, showing the overview with icons (left) and a notification pop-up (right).

informedness. Such a notification system could specifically benefit particular roles and tasks, such as the district police officer. Finally, by allowing officers to input information based on actions they performed, information sharing between colleagues can be more optimally supported. With an implemented LBNS, the following scenario would become reality for police officers on surveillance.

Police officer Fred walks on surveillance, equipped with a PDA with the LBNS. When he is some distance away from the playground area in his district, his PDA beeps. He checks the display and is informed that this is a criminal hotspot. Yesterday complaints were received about young people causing nuisance around the playground area. The notification further informs him that a colleague checked this location yesterday and sent the perpetrators away with a warning. Prompted by this notification, Fred checks the playground again. He does not encounter any problems and continues on his way. In a different street, he receives a notification that the resident of number 47 has a warrant for speeding. Based on this notification, Fred confronts the resident and succeeds in making an appointment for payment.

In short, using a mobile location-based notification system, officers will be better informed and aware of relevant locations in their vicinity as well as less reliant on remembering and accessing information at the right time and place.

\subsection{System Description}

Together with two commercial IT-companies, the Dutch police designed and implemented the LBNS on a PDA. The system was called Attentive Services ("Attendering Service" in Dutch). Five police officers with mobile computing experience were included in the design and implementation process as representative endusers.

The interface of the LBNS was designed as a geographical map application, showing relevant locations as icons ("points-ofinterest" or POI's) on this map (see Figure 1, left side). Exclamation marks were used as icons, showing different colors for different information sources (e.g. blue for agreements on location). In addition, the user's own location as well as the location of colleagues was indicated on the map. Possible map 


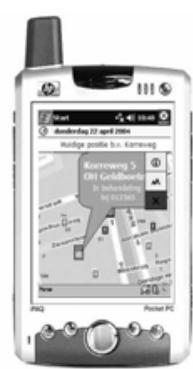

LBNS software on PDA platform
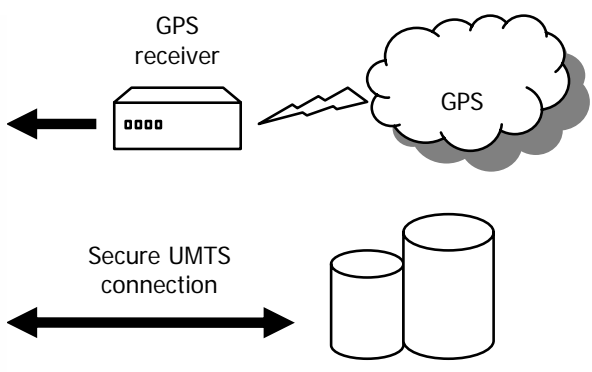

Police database P-info
Figure 2. System architecture and implementation

manipulations included zooming, panning and showing or hiding the POI's.

The LBNS was implemented on a Compaq IPAQ PDA. For an overview diagram of the system components, see Figure 2. The PDA was connected to the police database ( $P$-info) which contained the agreements, warrants and their addresses and details. The connection was established by means of a secured VPN tunnel over the UMTS data-network. In addition, the user carried a separate GPS device, connected to the PDA via Bluetooth. The LBNS coded the addresses to GPS coordinates and compared these coordinates to the current user location. When the user was within 40 meters of a POI location, the LBNS generated a notification.

A notification was presented as a salient sound signal and a small pop-up screen, pointing to the relevant location (see Figure 1, right side). The pop-up screen showed the address and perpetrator, and three buttons. By clicking on one of these, the user could decide to ignore the notification $(X)$, get more information $(I)$ or directly take action on the notification $(A)$. More information about the notification linked to details about the warrant or agreement in the police database. The "action-screen" provided users with opportunity for limited user feedback or notetaking. These notes could be read by colleagues. Finally, the officer could indicate whether the action was successful and the notification could be removed.

When multiple notifications appeared at the same address, these were presented in the same pop-up screen, indicating the total number of notifications. Users could switch back and forth between them using "previous" and "next" buttons. In addition to proactive notification, users could also click on icons on the map, to see what the agreement or warrant was about. Alternatively, they could access the police database on the PDA using a web browser.

A Kevlar protective casing was specially designed for this PDA. This casing allowed officers to attach the PDA directly to their belts, or let the PDA rest against their upper leg from a slightly longer attachment cable. This allowed them to view the display without having to detach the PDA from the belt.

\section{METHOD}

To test the efficiency, effectiveness and user experience of the LBNS for police officers, a longitudinal field evaluation was conducted over a period of four months. We were dependent on
Table 1. Research activities and number of participants (pp.) in the different phases of the field evaluation.

\begin{tabular}{|lll|}
\hline Phase & Research activities & \# of pp. \\
\hline Training (1 month) & Starting questionnaire & $\mathrm{N}=22$ \\
\hline \multirow{2}{*}{ Pre-test } & 1st Acceptance questionnaire & $\mathrm{N}=9$ \\
& Pre-interviews & $\mathrm{N}=8$ \\
\hline \multirow{2}{*}{ 1st Evaluation } & Usage questionnaire & $\mathrm{N}=26$ \\
(2 months) & Gathering log-files & $\mathrm{N}=26$ \\
Mid-test & Observation & $\mathrm{N}=2$ \\
\hline 2nd Evaluation & Usage questionnaire & $\mathrm{N}=11$ \\
(2 months) & Observation & $\mathrm{N}=14$ \\
\end{tabular}

the officers' response rate to the questionnaires as well as their availability for interviews

\subsection{Participants}

In total, thirty police officers participated in this study. Of this group, twenty-two (17 male, 5 female) participants filled out the starting questionnaire. Their mean age was 36.6 years $(\mathrm{SD}=7.6)$, with on average 12.2 years $(\mathrm{SD}=7.9)$ of police experience. Within this group, 4 participants were designated "district officers", whereas the rest had no specific role. All participants had elaborate experience in using desktop and mobile computers. Only three participants had never before used a mobile computer.

\subsection{Setting}

The evaluation took place in the district "Korrewegwijk" in the city of Groningen, the Netherlands. This district has a surface area of approximately $1.8 \mathrm{sq}$. kilometers (1.12 sq. miles) and 16.700 inhabitants ${ }^{1}$. The police department in this district employs 50 uniformed police officers.

The PDA with the LBNS was handed out to the thirty police officers. All users received one full day of training with the device. They were instructed on how to use it in their daily work practice (see Figure 3). After the training, they were asked to use the PDA for the whole evaluation period (four months).

\subsection{Measures}

This field evaluation employed a set of objective and subjective measures to establish users' expectations of the system, the experienced system accuracy, efficiency and effectiveness and the user experience.

Expectations. Users' expectations regarding the LBNS were established prior to the evaluation in interviews. Questions considered expectations about information delivery, work process support and success factors of the system.

Experienced system accuracy. The LBNS should provide relevant information correctly and at the right time and place. For example, receiving a notification about an address two blocks away, or that has already passed does not constitute high system accuracy. Thus, both the accuracy of the moment of notification

\footnotetext{
${ }^{1}$ Keyfigures available online at groningen.buurtmonitor.nl
} 

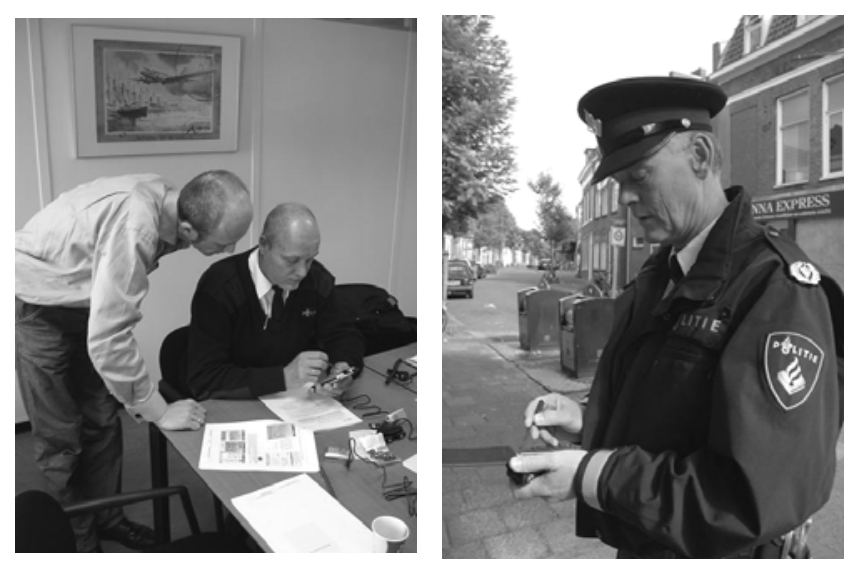

Figure 3. Police officers on training (left) and using the LBNS on surveillance (right).

presentation as well as the accuracy of the information itself is assessed using questionnaires, interviews and participatory observation.

Efficiency and effectiveness. Efficiency was measured with objective methods (usage data) and subjective methods (interviews and questionnaires). It is expressed as the subjective user judgements about their informedness, the relevance of the notifications and the influence of the system on the work processes. In addition, efficiency was regarded as the number of repetitions of notifications (how many identical notifications were given to each user). Effectiveness is measured as actual operational results based on the notifications (e.g. police officers undertook action or apprehended a person). Also, effectiveness was expressed as users' comments in the interviews and questionnaires.

User experience. The user experience of the LBNS consists of the user's appraisal of the ease with which they can work with the system and the usability of the interface. It was assessed whether police officers felt they could reach their goals with the system in a fast and efficient way. Too many interface operations, slowness, errors or inaccuracies tend to have a negative impact on the user experience. In addition, system complexity, system speed and physical comfort were investigated.

\subsection{Materials}

This study employed three different online questionnaires, two semi-structured interviews and log files that captured system events. The starting questionnaire asked about participants' age and police experience, use of (mobile) computers, email and internet. These interviews lasted approximately 30 minutes. The usage questionnaire consisted of 14 statements concerning the daily use of the LBNS. The answering options ranged from -3 "strongly disagree" to +3 "strongly agree". The statements dealt with the experienced support of the LBNS in the two work processes (agreements and warrants), the system performance and distraction or interruption by the notifications. The acceptance questionnaire consisted of 25 statements about support, trust and usability of the system. The same answering options were used as in the other questionnaire. In addition, four open questions regarding positive and negative aspects as well as possible improvements to the system. The semi-structured interviews contained questions regarding current work processes and users' expectations of the system. The $\log$ files recorded all notifications, user actions and system events on the PDA with a timestamp. From these files, usage data on notifications and user actions was calculated.

\subsection{Procedure}

The evaluation took place from April till August 2007. See Table 1 for a timeline of research activities. The evaluation period started with training with the system and filling out the starting questionnaire. After the training, the pre-interviews were held. Police officers used the LBNS in their daily work activities for the rest of the evaluation period. At two moments in the evaluation, a researcher accompanied the police officers on surveillance for participatory observation. Every week, users were asked to fill out the usage questionnaire. In addition, at two moments during evaluation, the acceptance questionnaire was filled out. All users received an email, prompting them to fill out the questionnaires online. Finally, at the end of the evaluation period, another round of interviews was held. The response rates to the questionnaires and the number of participants in each research activity is specified in the final column of Table 1.

\section{RESULTS}

All data was analyzed and frequencies or percentages were calculated. For selected questionnaire items, statistical significance was calculated using t-tests for a single mean to see whether the answer deviated from neutral.

\subsection{Expectations}

In interviews with 8 participants, prior to the evaluation, their expectations about the system were established. All participants expect to be better informed due to the LBNS, which they expected would result in higher efficiency and effectiveness of their work. Especially apprehending of persons and collecting open warrants should increase. In addition, the awareness of agreements on location and changes made by colleagues is expected to increase by using the system. They expect the system to specifically aid the district police officers and the "free shifts", but not the emergency response shift. These officers generally do not have the time to respond to notifications. Having information about colleagues' location seemed helpful to them as well.

Half of the participants expect system usability and stability to be critical success factors for user acceptance. In addition, the speed of the system is important as well as the accuracy; notifications have to be given at the right location. Two officers are concerned about distraction due to too many interruptions from the system.

\subsection{Usage data}

From the logfiles, usage data was analyzed from 24 users over a 55-day period. Only usage data was included from users who had received ten or more notifications during this time. In total, users received 3647 notifications, regarding 239 unique incidents. Further analysis showed that only four locations were responsible for 2566 notifications. These locations were exceptional institutions (such as a forensic psychiatric intstitution) that housed persons with multiple open warrants, resulting in 10 to 25 notifications at a time. To calculate the usage data more accurately, multiple notifications at these locations were counted as a single notification whenever they occurred. This filtering 
Table 2. Usage data over the first evaluation period

\begin{tabular}{|llrr|}
\hline Process & User action & Total & \% \\
\hline \multirow{2}{*}{ Notification } & Receive notification & 1658 & 100.0 \\
& Ignore notification $(X)$ & 140 & 8.4 \\
\hline & Requesting more information $(I)$ & 313 & 18.9 \\
& Take action $(A)$ & 105 & 6.3 \\
Handling & Nothing to report & 51 & 3.1 \\
Notification & Finished, do not remove & 16 & 1.0 \\
& Finished, remove & 6 & 0.3 \\
\hline Request & Agreements on location & 138 & 8.3 \\
\hline
\end{tabular}

resulted in 1658 notifications over the whole period. $94 \%$ of the notifications concerned open warrants, while the remaining $6 \%$ dealt with agreements on location.

The log files specified system events as well as user reactions (e.g. ignore, request more information, took action on the notification, remove notification at the location). Usage data (in frequencies and percentages) is presented in Table 2. The usage data showed that for most notifications, no response was given. It could not be determined whether the information in the notification was still received by the user or that the notification went unnoticed. Because the notification pop-up was presented directly in the display, the officer still could have noticed it, but not responded to it. Only 19\% of the total amount of notifications was followed up by requesting additional information. Action was undertaken on only $6 \%$ of the notifications (pressing the action button). Police officers often did not indicate the results of their action in the action screen. In $3 \%$ of the notifications, there was nothing to report (e.g. the perpetrator was not at home). Twentytwo $(16+6)$ specific instances were counted where the notification was handled with positive operational results; this represents $1.3 \%$ of the total number of notifications.

Average number of notifications was calculated per notification, per user and per 24 hour time period. On average, each unique notification was presented 5.7 times during the whole period. Each user received 69 notifications on average, with two users receiving more than 200 notifications over the whole period. Almost half of the participants received less than 50 notifications.

The log files showed approximately 4 active users per 24 hour period. During this period, on average 30.4 notifications were sent, although this number fluctuated heavily ( $\mathrm{SD}=21.3$ ). Most of the notifications were sent during the day shift ( $63 \%$ between 9:00 and 15:00). During the afternoon, evening and night shifts, respectively $20 \%, 8 \%$ and $9 \%$ of all notifications were generated. Although less officers are on shift during evening and nights, these numbers still show a preference for using the system during the daytime.

\subsection{Experienced system accuracy}

In general, users commented that the icons are represented in the interface accurately at the right location. It could not be determined if the notifications were always delivered at the right location. The questionnaires contained two statements concerning this issue; "Notifications are presented on the right moment" and
Table 3. Selected questionnaire items. Positive scores indicate agreement and negative scores indicate disagreement (* = significantly different from neutral at $\mathbf{p}<.05$ ).

\begin{tabular}{|lr|}
\hline Questionnaire item & Score \\
\hline Notifications were presented at the right moment & 0.11 \\
Notifications were presented too far away from the location & 0.38 \\
\hline Notifications often contained obsolete information & $\mathbf{0 . 5 4}$ \\
Notifications often are not relevant & $\mathbf{- 0 . 6 3 ^ { * }}$ \\
The system provided necessary information & 0.19 \\
Using the system, I can work faster & 0.28 \\
The system has a negative impact on my work & $\mathbf{0 . 6 6} *$ \\
Using the system, I can work more effectively & $\mathbf{0 . 6 9 *}$ \\
The system improved the handling of warrants & $\mathbf{0 . 6 2} *$ \\
The system improved my awareness of agreements & 0.44 \\
\hline The notifications were interruptive in my work & $\mathbf{0 . 5 5 ^ { * }}$ \\
The notifications often interrupted handling incidents & $\mathbf{0 . 5 7 ^ { * }}$ \\
The system presented too many notifications & 0.49 \\
\hline
\end{tabular}

"Notifications are presented too far from the location". Considering the neutral response to these statements (see Table 3 ), users apparently sometimes agreed and sometimes disagreed with these statements. During observation, most of the notifications were presented right before or at the address. However, some notable exceptions were observed as well, where notifications were received blocks from the actual address, back at the station or were not presented at all. Presumably, these exceptions were due to technical errors.

The accuracy of the information in the notification is also important. Users did not agree with the statement "Notifications often are not relevant", this effect was significantly different from neutral. However, in the questionnaires and interviews, users indicated that in general, too many notifications were delivered, they contained obsolete information, or they were repeated too often.

\subsection{Efficiency and effectiveness}

In the questionnaires and final interviews, users indicated to feel very positive about the concept of the LBNS. They regard their work to be more effective due to the system, but not more efficient. They are better informed of open warrants and agreements on location. However, all users indicate that the LBNS is an addition to their work, not a guiding principle. For example, they ignore notifications that are not directly relevant.

The notifications provide information clearly and quickly. In the questionnaires, users related 14 specific instances where the LBNS provided positive contribution to operational results (e.g. apprehension of persons). Also the opportunity to request additional information from police databases was considered very valuable. Finally, the LBNS showed the location of colleagues in the display. Users indicated that they did not use this information. This could be because of the relatively low number of users per day (4 on average) but also because police officers kept track of each others location via mobile phone or radio transceiver.

The downside of using the LBNS is that notifications can be interruptive or distracting during work. In the questonnaires, users 
indicated that notifications were often interruptive, especially during another incident or talking to people. As mentioned before, the system provided too many notifications (see Table 3). Users themselves indicated filtering of notifications (e.g. based on importance) as a solution to this problem.

\subsection{User experience}

Users mention that the LBNS is usable, understandable and clear, and that learing to work with the system is easy. However, there are three aspects that negatively influence the user experience of the system. First, the LBNS in its current form is a complex and vulnerable system, which relies heavily on the correct functioning of Bluetooth, WiFi and Virtual Private Network (VPN) connections. This lack of robustness results in occasional system malfunctions, while the complexity makes it hard for users to determine what went wrong and troubleshoot. Often, the only way to get the system up and running again is to reset the whole PDA. Second, the log in procedure is very cumbersome, requiring three different login name / password combinations, including a code that changes every 30 seconds (provided by a digital token). Third, the system is relatively slow. Reloading or refreshing the interface can take up to a minute, while system reactions to user actions can be very slow. This results in frustration on the users' part, because the system is not available when they need it.

The interface of the LBNS is positively commented on. The icons are clear, the three buttons I (information), A (action) and X (close) are clear and concise. Some users indicate that the buttons and text are too small. There is certainly more space available in the notification pop-up screens to enlarge the text and buttons.

The PDA itself is considered an extra burden to carry on the police belt. This belt is often completely filled with gun, pepperspray, gloves, handcuffs, etc. In the questionnaires, 12 users mention the PDA as physically "burdensome", and the separate GPS module as "unnecessary".

\section{DISCUSSION}

In this field evaluation, we described the use and implementation of a location-based notification system for police officers. Thirty police officers used the LBNS during four months in their daily work practice. Using questionnaires, interviews, usage data and observation, we evaluated the system's accuracy, the effects on efficiency and effectiveness of work processes and the user experience.

In presenting the notifications, the accuracy of the LBNS was variable. Notifcations were not always presented at the right location and the information is sometimes obsolete. The system accuracy might be improved by consequently presenting notifications at the right location. The system supports the work processes of open warrants and agreements on location; police officers are better informed and have necessary information about (prior) incidents available within the use context. This awareness led to positive operational results such as the apprehension of perpetrators.

Users consider the interface of the LBNS easy and usable to work with and the screen design clear and concise, despite their perceived complexity of the system. This complexity might be mitigated by integration of different system components (such as the separate GPS device).
The downsides are that the current system can be slow, the log-in procedure is cumbersome and the system is lacking robustness. These downsides led six participants to cease further participation before the end of the evaluation period. In addition, users consider the notifications often distracting or interruptive. This can be concluded from the answers to the questionnaires and in the usage data. Currently, only $18 \%$ of the notifications is followed up and $1 \%$ is acted upon and "solved". This might seem as a low number, but actual operational results are dependent on a number of factors. When a police officer wants to apprehend a person for an open warrant, as suggested by the notification, but no prison cells or police backup is available, it is considered not safe to proceed with action.

In its current implementation, the LBNS sends notifications to every police officer within the notification perimeter. Consequently, police officers often are already aware of the information in the notification, thus making it less relevant. In addition, not all notifications are relevant for every police officer. For example, district officers are very familiar with the agreements on location in their district. In contrast, police officers with the duty of emergency handling do not have the time to respond to open warrants. Finally, thought must be given on how to handle locations with 10 to 25 different notifications, such as special criminal institutions. These locations can be considered known to police officers, making it not necessary to notify them. These issues seriously affect the use of the system and must be addressed.

This field evaluation provided some valuable lessons on methodology. The quality of the results was dependent on the availability and response rate from the police officers. This made it impossible to conduct the pre and post interviews with exactly the same participants. The response rate on the weekly questionnaires was especially low in the second evaluation period, around $50 \%$. Participatory observation provided valuable insight into how the users used the system, but the officers were conscious that they were being observed. Finally, it proved difficult to quantify improvements in efficiency and effectiveness of the two work processes (agreements and warrants) that could be attributed to the system. Before the introduction of the notification system, efficiency and effectiveness were not recorded, making it hard to establish a baseline. One logical solution would be to measure on how many notifications action was taken and could subsequently be removed. However, agreements are necessary knowledge, but do not need to be removed, making this not a valid performance criterion for agreements. Number of removed notifications is not a valid performance criterion for warrants as well, because this depends very much on the situation whether a warrant can be afgehandled. The perpetrator can be not at home or there can be no room in the prison cells. In these cases, the LBNS and the user can have performed accurately, but still the action did not have the desired effect.

\subsection{Recommendations}

In its current implementation, the functional design of the LBNS is not appropriate for actual use in the police context. The downsides of the system (interruption, non-relevant notification) outweigh the positive operational results. Possible solutions might be intelligent filtering or personalization of notifications, 
integration of different system components and implementing the briefing focal points.

- Intelligent filtering. Filtering of notifications can lead to less interruption or distraction from other incidents. Notifications can be filtered based on incident priority, time of day or specific characteristics such as the height of the fine in the warrant. Furthermore, a personal user model can ensure that officers only receive notifications that are relevant for their task, role, availability or specific shift. Finally, the LBNS should keep track of who has already received a specific notification, and not notify this person again within some timeframe.

- Integration of different system components. Integration will lead to reduced system complexity, making it easier for the users to comprehend the system and troubleshoot when errors occur. For example, the separate GPS module can be integrated with the PDA, or the three different log-in procedures can be integrated into one.

- Implementing briefing focal points. This police information was not yet implemented in the current LBNS. As this information is more dynamic then either warrants or agreements, it is expected to be very relevant to include in the system. In addition, these focal points are more limited in number then warrants and agreements, so this is not expected to lead to increased interruptiveness.

\subsection{Conclusions}

This paper reported a longitudinal field study of a location-based notification system for mobile police officers. The LBNS supported the police procedures it was designed for, but in the current implementation caused too much interruption by non relevant notifications. Possible solutions include intelligent filtering of notifications based on user and context characteristics, integration of system components and also notifying briefing focal points. The conclusion from this evaluation is that the added benefit of the LBNS lies in proactively informing police officers to relevant information in their vicinity. However, the mitigation of interruption due to irrelevant notifications must be a focal point in the redesign of this innovative mobile system. By taking into account the proposed recommendations, location based notification can fulfill its potential added benefit for police officers on surveillance.

\section{ACKNOWLEDGEMENTS}

This research was carried out within the MultimediaN project, funded by the Dutch Department of Economic Affairs. We would like to acknowledge Roy Mente, Elle de Jonge and Jan van de Kooij for their help in the evaluation. Special thanks to the police officers and management of the Groningen Police Department.

\section{REFERENCES}

[1] Baber, C., Haniff, D., Sharples, M., Boardman, M., \& Price, A. (2001). A requirements analysis of personal mobile computers for police officers. In D. Harris (Ed.), Engineering Psychology and Cognitive Ergonomics, Volume Six (pp. 2128). Aldershot: Ashgate.
[2] Cheverst, K., Mitchell, K., \& Davies, N. (2002). Exploring Context-aware Information Push. Personal and Ubiquitous Computing, 6, 276-281.

[3] Consolvo, S., Harrison, B., Smith, I., Chen, M., Everitt, K., Froehlich, J., \& Landay, J.A. (2007) Conducting In Situ Evaluations for and With Ubiquitous Computing Technologies. International Journal of Human-Computer Interaction, 22, (1-2), 103-118.

[4] Dunham, R.G. and Alpert, G.P. Critical Issues in Policing: Contemporary Readings. Waveland Press (1989).

[5] Jiang, X., Chen, N., Hong, J., Wang, K., Takayama, L., \& Landay, J. (2004). SIREN: Context-aware Computing for Firefighting. In Proceedings Pervasive.

[6] Kjeldskov, J., Skov, M. B., Als, B. S., \& Hoegh, R. T. Is it worth the hassle? Exploring the added value of evaluating the usability of context-aware mobile systems in the field. In Proceedings of Mobile HCI 2004, Glasgow, Scotland. (2004).

[7] Munson, J. P. and Gupta, V. K. Location-based notification as a general-purpose service, in Proceedings of the 2nd International Workshop on Mobile Commerce (WCM '02), ACM Press, 2002, 40-44.

[8] Murray, J. "Wearable Computers in Battle: Recent Advances in the Land Warrior System," iswc, p. 169, Fourth International Symposium on Wearable Computers (ISWC'00), 2000

[9] Nulden, U. Investigating police patrol practice for design of IT. In Extended abstracts of the CHI 2003 (pp. 820-821). Washington, DC: ACM (2003).

[10] Streefkerk, J.W., Van Esch-Bussemakers, M.P., Neerincx, M.A. \& Looije, R. Evaluating Context-Aware Mobile Interfaces for Professionals. In J. Lumsden (Ed.), Handbook of Research on User Interface Design and Evaluation for Mobile Technology. IDEA group. (2008)

[11] Sampat, M., Kumar, A., Prakash, A., McCrickard, S. Increasing Understanding of a New Environment using Location-Based Notification Systems. Poster paper in Proceedings of 11th International Conference on HumanComputer Interaction (HCII '05), Las Vegas NV, July 2005

[12] Te Brake, G., de Greef, T., Lindenberg, J., Rypkema, J. and Smets, N.. Developing adaptive user interfaces using a game-based simulation environment. In Proc. ISCRAM, (2006).

[13] Uluca, D., Streefkerk, J.W., Sciacchitano, B. and McCrickard, D.S. Designing Automated Handheld Navigation Support. Accepted for Mobile HCI 2008, Amsterdam, the Netherlands (in press).

[14] Van Eijk, R., de Koning, N., Steen, M. \& Reitsma, E. Developing a We-Centric Service for Mobile Police Officers to Support Spontaneous Communication. Technical report Enschede: Freeband, 2006. Available from: https://doc.freeband.nl/dscgi/ds.py/Get/File-67631 